\title{
A Rigorous Proof for the Strong Goldbach Conjecture
}

\author{
Abdelilah Aouessare \\ Abdelmalek Essaadi University \\ Faculty of Science \\ Tetuan, Morocco
}

\author{
Abdeslam El Haddouchi \\ Abdelmalek Essaadi University \\ Faculty of Science \\ Tetuan, Morocco
}

\author{
Mohamed Essaaidi \\ Mohammed V University \\ ENSIAS \\ Rabat, Morocco
}

\begin{abstract}
In this paper, a rigorous proof of the strong Goldbach Conjecture is provided. This proof is mainly based on a very special expression of even numbers we propose and which takes the form of $(2 n+6)$. Even numbers expressed in this form can be expressed as sums of two primes of the form of $(3+2 i)$ and $(2 n+3-2 i)$, where I and $n$ are positive integers. We show that it is always possible to find at least one pair of prime numbers according to the former two expressions for any given even number greater or equal to 6 . Moreover, the prime numbers theorem is used to investigate the number of primes' pairs corresponding to even numbers. The obtained results showed that all even numbers have at least one pair of primes verifying this conjecture. Thus, this result provides a rigorous proof for Goldbach conjecture which is still considered, to our best knowledge, among the open problems of mathematics.
\end{abstract}

\section{General Terms}

Number theory, Prime numbers, Algorithms.

\section{Keywords}

Goldbach conjecture, Numbers theory, prime numbers, even numbers, proof.

\section{INTRODUCTION}

In the letter sent by Goldbach to Euler in 1742 [1] he stated that "Its seems that every odd number greater than 2 can be expressed as the sum of three primes". Note that Goldbach had considered 1 to be a prime number, a convention that was abandoned later on. As reformulated by Euler, an equivalent form of this conjecture called the "strong" or "binary" Goldbach conjecture states that all positive even integers greater or equal to 4 can be expressed as the sum of two primes which are sometimes called a Goldbach partition.

Many mathematicians and amateurs tried to provide a proof for this conjecture during the last couple of centuries. Some approaches proposed in for this purpose can be found in references [2-11]. Though the ternary conjecture seems to have been rigorously proved in 2013 by Helgofft [11], the strong or binary conjecture is still today an open problem despite several attempts using different approaches.

In this paper, we propose a proof of the strong Goldbach conjecture based on a very interesting approach whose key foundation is a general form for even numbers greater or equal to 6 we propose, namely, $(2 n+6)$ which we develop as a sum of two prime numbers. According to this expression, it is always possible to find at least one pair of prime numbers whose sum gives the even number in question, verifying thus, Goldbach conjecture. Prime numbers theorem is then used to analyze the behavior of number of primes' pairs satisfying this conjecture.
As a consequence of this analysis, it is concluded that Goldbach conjecture is rigorously proved and that it should be reformulated as follows:

" Every even number greater than 2 have at least one pair of primes such that it is equal to their sum.".

We ask that authors follow some simple guidelines. In essence, we ask you to make your paper look exactly like this document. The easiest way to do this is simply to download the template, and replace the content with your own material.

\section{GOLDBACH CONJECTURE AND EVEN NUMBERS REPRESENTATION}

\subsection{Goldbach conjecture}

One can easily verify, as illustrated in the examples below, that every even number can involve at least one pair of primes satisfying Goldbach conjecture. To this end, we prefer to reformulate this conjecture as follow: "Every even number greater than 2 have at least one pair of primes such that it is equal to their sum.". Let's consider $(p, q)$ the pair of primes such that $\mathrm{p} \leq \mathrm{q}$ and the even number $2 \mathrm{n}=\mathrm{p}+\mathrm{q}$, where $\mathrm{n}$ is an integer, $\mathrm{n}>1$.

Examples:

For the sake of illustration, let's consider the even numbers 16,22 and 42 which can be written according to Goldbach conjecture as follow:

$$
\begin{aligned}
& 16=3+13=5+11 \\
& 22=3+19=5+17=11+11 \\
& 42=5+37=11+31=13+29=19+23
\end{aligned}
$$

Therefore, 16 has 2 pairs of primes verifying Goldbach conjecture, 22 has 3 pairs and 42 has 4 pairs.

\subsection{Even numbers Representation}

Let's consider even numbers of the form $(2 n+6)$, where $n$ is a positive integer number $(n \geq 0)$. In this case, we can state a modified Goldbach conjecture as follows:

Every even number of the from $(2 n+6)$ can be written as the sum of two primes such that

$2 n+6=(3+2 i)+(2 n+3-2 i)$

where $n$ and $i$ are positive integers $(n, i \geq 0)$ such that $(3+2 i)$ and $(2 n+3-2 i)$ are prime numbers.

So, as we will see in the examples below, it is always possible to find values for $i$ and $n$ such that $(3+2 i)$ and $(2 n+3-2 i)$ are prime numbers.

Though expression (1) starts with the even number 6 , it is possible to generalize the proof for the even number 4 for which Goldbach conjecture is straightforward. 


\section{Examples}

$$
\begin{array}{rr}
\text { For } n=0 \text { and } i=0: & 6=3+3 \\
n=1 \text { and } i=0: & 8=3+5 \\
n=2 \text { and } i=0: 10=3+7 \\
n=3 \text { and } i=0: 12=3+9,
\end{array}
$$

Since 9 is not prime, we increment $i(i=1)$, which gives $12=$ $5+7$

$$
\mathrm{n}=4 \text { and } \mathrm{i}=0: \quad 14=3+11
$$

$$
\begin{array}{ll}
\mathrm{n}=5 \text { and } \mathrm{i}=0: & 16=3+13 \\
\mathrm{n}=6 \text { and } \mathrm{i}=0: & 18=3+15 .
\end{array}
$$

\begin{tabular}{|c|c|c|c|c|c|c|c|c|c|c|c|c|c|c|}
\hline & 3 & 5 & 7 & 9 & 11 & 13 & 15 & 17 & 19 & 21 & 23 & …………… & $2 n+3$ & $\begin{array}{c}\text { Number primes' } \\
\text { pairs }\end{array}$ \\
\hline 6 & 3 & & & & & & & & & & & & & 1 \\
\hline 8 & 5 & 3 & & & & & & & & & & & & 1 \\
\hline 10 & 7 & 5 & 3 & & & & & & & & & & & 2 \\
\hline 12 & 9 & 7 & 5 & 3 & & & & & & & & & & 1 \\
\hline 14 & 11 & 9 & 7 & 5 & 3 & & & & & & & & & 2 \\
\hline 16 & 13 & 11 & 9 & 7 & 5 & 3 & & & & & & & & 2 \\
\hline 18 & 15 & 13 & 11 & 9 & 7 & 5 & 3 & & & & & & & 2 \\
\hline 20 & 17 & 15 & 13 & 11 & 9 & 7 & 5 & 3 & & & & & & 2 \\
\hline 22 & 19 & 17 & 15 & 13 & 11 & 9 & 7 & 5 & 3 & & & & & 3 \\
\hline 24 & 21 & 19 & 17 & 15 & 13 & 11 & 9 & 7 & 5 & 3 & & & & 3 \\
\hline 26 & 23 & 21 & 19 & 17 & 15 & 13 & 11 & 9 & 7 & 5 & 3 & & & 3 \\
\hline & & . & . & . & & . & 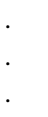 & & . & . & . & . & & \\
\hline $\begin{array}{l}0 \\
\stackrel{+}{\Sigma} \\
\text { L }\end{array}$ & 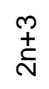 & 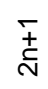 & $\overline{\grave{N}}$ & $\begin{array}{l}\text { m } \\
\stackrel{1}{N}\end{array}$ & $\begin{array}{l}\stackrel{p}{\perp} \\
\text { N }\end{array}$ & $\hat{i}$ & $\stackrel{\text { i }}{\stackrel{N}{N}}$ & $\frac{\overline{1}}{\frac{1}{N}}$ & $\frac{m}{\frac{m}{N}}$ & $\frac{10}{\dot{L}}$ & $\frac{N}{\frac{1}{c}}$ & & 3 & $\mathrm{~Np}$ \\
\hline
\end{tabular}

Since 15 is not prime we take $\mathrm{i}=1$, and we get $(5,13)$.

Therefore, equation (1) is the key foundation for the proof we propose for the strong Goldbach conjecture.

Table 1 Goldbach conjecture table

\section{GOLDBACH CONJECTURE TRIANGLE}

The primes' pairs triangle for a given even number $\mathrm{N}$ is obtained from a table where the first row is made of odd numbers of the form $(2 n+3)$ and the first column is made of even numbers of the form $(2 n+6)$, where $n$ is a positive integer $(n \geq 0)$. Then, the boxes $(i, j)$ of this table are filled in by subtracting the content of $(1, j)$ boxes from those of $(i, 1)$ where $i, j>1$. The boxes for which the obtained result is a prime are indicated in grey color and boxes for which it is negative or less than 3 are kept empty as shown in Table 1 below illustrating Goldbach conjecture triangle. This triangle

indicates the primes' pairs verifying Goldbach conjecture for a given even number greater or equal to 6 . The last column of this table gives the number of primes' pairs corresponding to the even numbers of the first column.

The cumulative total number of primes' pairs (i.e. Np) corresponding to all even numbers up to $(2 \mathrm{~N}+6)$ is obtained by summing up all the elements of the last column. From the data presented in this table; it is easily seen that even numbers have at least one primes' pairs satisfying Goldbach conjecture.

\section{CUMULATIVE NUMBER OF PRIMES' PAIRS}

In order to calculate the number of primes' pairs corresponding to an even number, let's consider the Prime Number Theorem [12]. This is the most fundamental theorem of prime numbers which defines $\pi(\mathrm{N})$ the prime counting function giving the number of primes smaller than an even number $\mathrm{N}$ as follows

$$
\pi(\mathrm{N}) \approx \mathrm{N} / \operatorname{Ln}(\mathrm{N})
$$

This is an approximation of this number which improves as $\mathrm{N}$ increases. A better approximation of this number is obtained from a function called the logarithmic interval as follows.

$$
\pi(\mathrm{N}) \approx \operatorname{Li}(\mathrm{N})=\int_{2}^{\mathrm{N}} \mathrm{dx} / \operatorname{Ln}(\mathrm{x})
$$

However, Riemann function is even more accurate compared to the former two expressions.

The total number of primes' pairs verifying Goldbach conjecture for an even number $\mathrm{N}$ can be obtained in the same manner. To this end, let's analyze in Table 2 the behavior of this number which is equal at least to 1 (one pair of primes verifying the conjecture). Moreover, only half of the interval $(3,2 \mathrm{~N}+3)$ contributes to the total number of primes' pairs 
corresponding to even numbers smaller than the even number $\mathrm{N}$. Therefore, this number can be expressed as indicated below:

$$
\mathrm{L}(\mathrm{N})=\pi(2 \mathrm{~N}+3) / 2) \times \pi(2 \mathrm{~N}+3) / 2
$$

Taking into account the expression of $\pi(\mathrm{N})$ given in (2) we obtain:

$$
\mathrm{L}(\mathrm{N})=(\mathrm{N} / 2)^{2} /(\operatorname{Ln}(\mathrm{N} / 2) \operatorname{Ln}(\mathrm{N}))
$$

\section{Examples:}

For $\mathrm{N}=26, \quad \mathrm{~L}(26)=20.31$ which is not very close to the exact cumulative total number of primes' pairs of 22 . The discrepancy between the exact and estimated number using equation (5) may be explained by the fact that prime number theorem precision increases with increasing values of $\mathrm{N}$.

In Table 2 we can see an illustration of the evolution of the cumulative number of primes for even numbers $10^{2}, 10^{3}, 10^{4}$, $10^{5}$ and $10^{6}$.

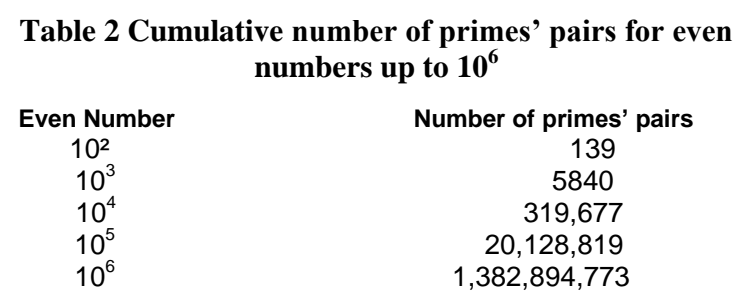

It is clear from these examples that the total number of primes' pairs corresponding to even numbers less than $10 \mathrm{k}$ increases significantly with $\mathrm{k}$.

The number of primes' pairs verifying Goldbach conjecture corresponding to a single even number N1 is obtained according to the following equation:

$$
\mathrm{Np}=\mathrm{L}(\mathrm{N} 1)-\mathrm{L}(\mathrm{N} 2)
$$

where $\mathrm{N} 2$ is an even number such that $\mathrm{N} 1-\mathrm{N} 2=2$.

$\begin{array}{crc}\mathbf{N} & \mathbf{N p} & \mathbf{N p} \text { (exact) } \\ 10^{1} & 1.2 & 2 \\ 10^{2} & 4.23 & 6 \\ 10^{3} & 19.72 & 20 \\ 10^{4} & 127.42 & 127 \\ 10^{5} & 802.95 & - \\ 10^{6} & 5519.06 & -\end{array}$

From the examples presented above it is clearly seen that equation (6) is a very good approximation of the number of primes' pairs corresponding to a given even number, especially, for even numbers greater than or equal to 103. It is also clear that for every even number there is at least one pair of primes verifying Goldbach conjecture.

From here we can confirm that the strong Goldbach conjecture is true and that it should be reformulated as follows:
"There is at least one pair of primes such that a positive even integer greater than or equal to 4 can be expressed as their sum".

\section{CONCLUSIONS}

We have provided a rigorous proof of the strong Goldbach Conjecture which is mainly based on a very special expression of even numbers we propose, namely, $(2 n+6)$. Even numbers expressed in this form can be expressed as sums of two primes of the form of $(3+2 i)$ and $(2 n+3-2 i)$, where $I$ and $n$ are positive integers. We have shown that it is always possible to find at least one pair of prime numbers according to the former two expressions for any given even number greater or equal to 6 . Since the even number 4 already verifies Goldbach conjecture, we can state that all even numbers greater or equal to 4 can be expressed of a sum of two primes. Furthermore, a given even number may have more than one pair of primes satisfying Goldbach conjecture.

\section{REFERENCES}

[1] Christian Goldbach, Letter to L. Euler, June 7 (1742).

[2] Yuan Wang, Goldbach Conjecture, 2nd edn., World Scientific Publishing, New Jersey, Singapore (2000).

[3] Richard K. Guy, Unsolved problems in number theory, Third Edition, Springer-Verlag, Berlin (2004).

[4] Nikolai G. Chudakov, On the Goldbach problem, Doklady Akademii Nauk SSSR, 17 (1937), 335-338.

[5] T. Estermann, On Goldbach's problem: proof that almost all even positive integers are sums of two primes , Proc. London Math. Soc., S' er. 2, 44 (1938), 307-314.

[6] Matti K. Sinisalo, Checking the Goldbach Conjecture up to $4 \cdot 1011$, Math- ematics of Computation, 61, No. 204 (1993), 931-934.

[7] J. R. Chen, On the representation of a larger even integer as the sum of a prime and the product of at most two primes, Sci. Sinica, 16 (1973), 157-176.

[8] J org Richstein, Verifying the Goldbach conjecture up to 4 - 1014, Mathematics of Computation, 70, No. 236 (2000), 1745-1749.

[9] Neil Sheldon, A statistician's approach to Goldbachs Conjecture, Teaching Statistics 25, No. 1 (2003), 12-13.

[10] Song Y. Yan, A simple verification method for the Goldbach conjecture, International Journal of Mathematical Education in Science and Technol- ogy, 25, No. 5 (1994), 681-688

[11] H. A. Helfgott, "The Ternary Golbach Conjecture is True", arXiv:1312.7748v2 [math.NT], 17 Jan 2014.

[12] D. J. Newman, Simple Analytic Proof of the Prime Number Theorem, The American Mathematical Monthly, 87, No. 9 (1980), 693-696. 